Article

\title{
Lung Ultrasound in Children with Cystic Fibrosis in Comparison with Chest Computed Tomography: A Feasibility Study
}

\author{
Ioana Mihaiela Ciuca ${ }^{1}$ (D) Liviu Laurentiu Pop ${ }^{1}$, Mihaela Dediu ${ }^{1}$ (D), Emil Robert Stoicescu ${ }^{2,3}$ (D), \\ Monica Steluta Marc ${ }^{4, *(D)}$, Aniko Maria Manea ${ }^{5}$ and Diana Luminita Manolescu ${ }^{2}$ (D)
}

1 Pediatric Department, "Victor Babes" University of Medicine and Pharmacy Timisoara, Eftimie Murgu Square No. 2, 300041 Timisoara, Romania; ciuca.ioana@umft.ro (I.M.C.); liviupop@umft.ro (L.L.P.); dediu.mihaela@umft.ro (M.D.)

2 Radiology Department, "Victor Babes" University of Medicine and Pharmacy Timisoara, Eftimie Murgu Square No. 2, 300041 Timisoara, Romania; stoicescu.emil@umft.ro (E.R.S.); dmanolescu@umft.ro (D.L.M.)

3 Research Center for Pharmaco-Toxicological Evaluations, "Victor Babes" University of Medicine and Pharmacy Timisoara, Eftimie Murgu Square No. 2, 300041 Timisoara, Romania

4 Pulmonology Department, "Victor Babes” University of Medicine and Pharmacy Timisoara, Eftimie Murgu Square No. 2, 300041 Timisoara, Romania

5 Neonatology and Puericulture Department, "Victor Babes" University of Medicine and Pharmacy, Eftimie Murgu Square No. 2, 300041 Timisoara, Romania; manea.aniko@umft.ro

* Correspondence: marc.monica@umft.ro; Tel.: +40-721434044

check for

updates

Citation: Ciuca, I.M.; Pop, L.L.;

Dediu, M.; Stoicescu, E.R.; Marc, M.S.;

Manea, A.M.; Manolescu, D.L. Lung Ultrasound in Children with Cystic Fibrosis in Comparison with Chest Computed Tomography: A

Feasibility Study. Diagnostics 2022, 12, 376. https://doi.org/10.3390/

diagnostics12020376

Academic Editors: Marcello Demi and Gino Soldati

Received: 20 December 2021

Accepted: 30 January 2022

Published: 1 February 2022

Publisher's Note: MDPI stays neutral with regard to jurisdictional claims in published maps and institutional affiliations.

Copyright: (C) 2022 by the authors. Licensee MDPI, Basel, Switzerland. This article is an open access article distributed under the terms and conditions of the Creative Commons Attribution (CC BY) license (https:// creativecommons.org/licenses/by/ $4.0 /)$.

\begin{abstract}
Background: Cystic fibrosis (CF) lung disease determines the outcome of this condition. For lung evaluation processes, computed tomography (CT) is the gold standard, but also causes irradiation. Lately, lung ultrasound (LUS) has proven to be reliable for the diagnosis of consolidations, atelectasis, and/or bronchiectasis. The aim of our study was to evaluate the value of a newly conceived LUS score by comparing it to the modified Bhalla CT score. A further aim was to evaluate the correlation between the score and the lung clearance index (LCI). Methods: Patients with CF were screened by LUS, followed by a CT scan. Spearman's test was used for correlations. Results: A total of 98 patients with CF were screened, and 57 were included in the study; their mean age was $11.8 \pm 5.5$ (mean $\pm \mathrm{SD}$ ) years. The mean LUS score was $5.88 \pm 5.4 \mathrm{SD}$. The LUS CF score had a very strong correlation with the CT score of $\mathrm{rs}=0.87(p=0.000)$. LUS showed a good sensibility for detecting atelectasis $(\mathrm{Se}=83.7 \%)$ and consolidations $(\mathrm{Se}=94.4 \%)$. A lower Se $(77.7 \%)$ and Sp $(9 \%)$ were found for cylindrical bronchiectasis. Conclusion: Our study shows that LUS and the lung CF score are parameters that can be used with a complementary role in the diagnosis and monitoring of CF lung disease in children.
\end{abstract}

Keywords: lung ultrasound; cystic fibrosis; computed tomography comparison; CT

\section{Introduction}

Cystic fibrosis is a complex disease characterized by significant clinical polymorphism, a special evolution, and severe complications that raise problems in the individual monitoring and management of the disease [1]. The pulmonary condition remains the most important issue that dictates the prognosis of the disease [2]. Therefore, an early diagnosis of pulmonary complications and the preservation of the lung function are essential. For the diagnosis and monitoring of CF lung disease, many investigations are used, from widely accessible chest X-ray examinations (CXR) [3]—which have lower sensitivity - to computed tomography (CT), the current gold standard [4]. HRCT is very sensitive for the detection of any structural changes, but its repeatability is restricted because of its significant irradiating potential. On the other hand, lung ultrasound is a non-irradiating, easy-to-use method, reliable for the detection of severe childhood pulmonary diseases, from pneumonia [5] to 
pulmonary fibrosis [6]; therefore, it is important to evaluate its potential in the accurate detection of CF lung disease.

Presently, lung ultrasound has demonstrated efficiency in the diagnosis of child pneumonia $[5,7]$ and other frequent childhood diseases, such as bronchiolitis, pneumothorax, atelectasis [8], pleural effusion, and pulmonary contusion [9]. It is important to note that lung ultrasound is more sensitive for the detection of smaller lesions [10]. LUS is also valuable for the examination of children diagnosed with tuberculosis, as it seems to be more sensitive than CXR [11]—especially for sickle cell disease [12], or rare diseases such as NEHI [13].

Studies have shown that LUS value in CF exacerbations [14] correlates with lung function tests [15], and reliable correlations between LUS and Chrispin-Norman X-ray score [16] or modified Bhalla CT score have been published [17].

The progression of structural lung deterioration requires objective measurements, such as CT scores [18-21], which offer the necessary support for correct monitoring and the possibility of a follow-up-a mandatory procedure for accurate lung evaluation.

Numerous lung ultrasound scores were developed as consistent, non-invasive tools for numerous diagnoses, such as respiratory COVID-19 in adults [22-24], pneumonia in elders [25], ARDS [26], and lung recruitment ventilation [27,28], with significant practical results. Similarly, for children's respiratory pathology, important achievements have been made by the use of LUS scores to evaluate COVID-19 pneumonia in neonates, pneumonia in children [29], and to predict the need for surfactants in neonates [30], or ventilation requirements in neonatal respiratory distress syndrome [31].

Therefore, in this study, we aimed to assess the value of a newly conceived lung ultrasound (LUS) score by comparing it to the HRCT modified Bhalla score, and to evaluate the correlation between the LUS score and the lung function expressed by the lung clearance index (LCI), which is the most accurate parameter for CF lung function evaluation [32,33].

The LUS scoring system is useful for the detection of multiple respiratory diseases, including features that can be found in CF, in addition to pneumonia [34] or COVID-19 [24].

Chest CT is the gold standard for the structural evaluation of CF lung disease, and the need of an objective marker led to creation of CT scores, which are able to estimate the severity and degree of the specific features that appear in CF [33]. The modified Bhalla score is an accurate and feasible way to assess the severity of $\mathrm{CF}$-a lung parenchymal disease-as it is closely correlated with lung functions [33], severe genotype, and chronic Pseudomonas infections [32,33,35]. In the study conducted by Leung A. et al. [35], modified Bhalla score included the evaluation of the presence, severity, and extent of bronchiectasis, bronchial wall thickening, mucus plugging, atelectasis/consolidation, and air trapping, using a 0-3 severity scale [35], thus simplifying the original Bhalla scale [20].

Even CT scores are used as a surrogate outcome in the evaluation of cystic fibrosis lung disease. In terms of sensitivity, it seems that LCI is comparable with CT. LCI is an indicator of irregular ventilation distribution that sensibly detects abnormal lung structure and early changes in the lungs with CF [32,33]; its practical usefulness lies in its applicability in younger children who cannot be subjected to spirometry, as only tidal breathing is necessary for performing multiple-breath washout (MBW) - the method through which LCI is obtained [32]. Several studies have demonstrated that LCI is better correlated with $\mathrm{CT}$ scores than spirometry parameters, and is very sensitive for the detection of early changes in CF lungs [32,33], suggesting that "LCI may be even more sensitive than HRCT scanning for detecting lung involvement in $\mathrm{CF}^{\prime \prime}$ [32].

Therefore, we conclude that the comparison between our new LUS-CF score and a CT examination - the gold standard for structural CF changes—and LCI-the most accurate lung function parameter-is the right premise for this feasibility study. 


\section{Materials and Methods}

\subsection{Study Population}

The study population was aged between 6 months and 18 years, diagnosed with typical cystic fibrosis and monitored at our CF center. They were invited to participate in the study, starting from October 2016 until March 2020. The study was approved by the Ethics Committee of the Clinical County Hospital (no.8/2016).

Each parent and, in cases over 12 years old, each child, signed the informed consent agreement regarding the agreement to participate in the study, in accordance with the Declaration of Helsinki.

\subsection{Study Protocol}

\subsubsection{Lung Ultrasound}

LUS was performed at the first clinical evaluation, before the biological tests and the computer scanning.

We used an Alpinion E-CUBE 9 ultrasound system, scanning with a linear probe of 7-12 MHz frequency and a 3.5-5 MHz convex probe, corresponding to the thoracic wall dimensions. The lung ultrasound was performed by a pediatric pulmonologist with 7 years of experience in LUS, blinded to previous CT examinations, and the stored images were checked by a senior radiologist with 8 years of expertise in lung ultrasound.

Most of the compliant children were evaluated in supine and prone positions, and then in their mothers' arms or in an upright position, if pleurisy was detected, for volume estimation.

The ultrasound evaluation protocol included scanning of the lung areas by longitudinal sections: right and left parasternal, medio-clavicular, anterior and posterior axillary, posterior by paravertebral, medio-scapular and posterior axillar lines. Moreover, the probe transversally scanned each intercostal space, in addition to the transabdominal approach through the liver and spleen window for costal diaphragmatic angles and retrocardiac consolidations. Separately, the hemithorax was virtually divided into 6 areas: 2 anterior-anterosuperior and anteroinferior; 2 lateral-superior and inferior lateral; and 2 posterior-superior and inferior $[6,34]$. The splenic ultrasound window was also used to evaluate the lower lobes of the left lung and the left costodiaphragmatic angle, as well as the hepatic window for the lower right lung artefacts.

LUS-CF scores were quantified as normal (0-1), mild (2-6), moderate (6-10), or severe $(>10)$.

\subsubsection{CT}

After the LUS examinations were performed, the patients underwent a CT scan every two years, as part of their regular evaluation, according to our national standards.

The CT scans were performed with a Philips MX 16 EVO 16-slice CT with dedicated pediatric protocols and a Neusoft NeuViz 16 Essence 16-slice CT in the Radiology Department of "Pius Brînzeu" County Emergency Clinical Hospital, Timisoara. The CT scans were optimally performed at $120 \mathrm{kVp}$, according to international standards. CT scans were acquired at a $2.5 \mathrm{~mm}$ and $5 \mathrm{~mm}$ slice thickness, with reconstruction images at $1.25 \mathrm{~mm}$. The images were stored in the workstation, the hospital's PACS system, and on CDs, and were interpreted by an experienced radiologist with CT competence and 16 years of experience.

The CT images were analyzed and scored using a modified Bhalla cystic fibrosis score for HRCT [35]. For evaluating the lung lesions, the score considered the quantification of the injuries to the respiratory tract: type (cylindrical, varicose, and saccular) and extension of bronchiectasis, the thickening of the bronchial walls in different stages (mild, moderate, and severe) and the extent of mucus plugging. The quantification of air-trapping zones and the extension of the lung parenchymal lesions (a consolidation area with air bronchogram, consolidation zones, and atelectasis) were also examined.

The scores were achieved for all five lung regions corresponding to the right upper, middle, and lower lung zones, as well as the left upper and left lower lung zones. The 
final lesion score was obtained by summing up the five-lobe score. According to the total severity score, CT scores were classified as mild (0-33), moderate (34-66), or severe (>66) [35].

\subsubsection{Lung Function}

Spirometry was performed in all patients over the age of 5 years old, as part of the biannual or 3-monthly evaluation, according to their age and infection status. The standards imposed by the American Thoracic Society/European Respiratory Society [36], along with the Global Lung Function Initiative 2012 reference equations [37], were used to calculate the percentage of the predicted parameter values, using a CareFusion machine.

LCI obtained by tidal breathing and by multiple-breath nitrogen (N2) washout was determined using Quark PFT (COSMED, Italy). The LCI was calculated as the number of lung volume turnovers (i.e., the cumulative expired volume divided by the functional residual capacity) needed to lower the end-tidal tracer gas concentration below $2.5 \%(1 / 40$ of starting level), with the normal values considered below 7 [38].

\subsection{Statistical Analysis}

For the descriptive statistics, the percentage values for categorical variables and continuous variables were expressed as the mean \pm standard deviation. The ShapiroWilk test was used to establish the distribution of our quantitative data. The data were investigated using IBM SPSS Statistics version 26.0. Spearman's correlation coefficient was used for the evaluation of the relationships between the quantitative variables. The coefficient Spearman's rho $<0.2$ was significant for showing the lack of relationship between the variables. The correlation was considered weak if Spearman's rho was between 0.2 and 0.29 , moderate with Spearman's rho 0.3-0.39, strong relationship if Spearman's rho had a value in the 0.4-0.67 interval, and very strong when Spearman's rho > 0.7 [39]. The KruskalWallis test was applied to compare the medians between the groups. The specificity and sensitivity rates were calculated, in addition to the positive and negative predictive values, while $p$-values were considered significant if $p<0.005$.

\section{Results}

\subsection{Descriptive Data}

A total of 98 patients with CF were screened, and 57 were included in the study, as CT was performed at their biannual evaluation. Their mean age was $11.8 \pm 5.5 \mathrm{SD}$ years (ranging between 3.3 and 21.8 years old), and $42.1 \%$ were females. Most of the patients had a severe genotype, almost half of them (49.1\%) being f508 del homozygous; the $\mathrm{f} 508$ del allele was present in $71.05 \%$ of the cases, followed by G542X in $6.1 \%$. The percentage of chronically infected patients was $=49.12 \%$ (33.3\% with Pseudomonas aeruginosa $15.7 \%$ with Staphylococcus strains, and 7\% were polymicrobial).

\subsection{LUS CF Score}

The artefacts used to define the pathological elements were as follows (Table 1$)$ : the presence of $\mathrm{A}$ lines - normal aspect $=0$ points; less than $3 \mathrm{~B}$ lines, thin $(<2 \mathrm{~mm}$ in width) $/$ intercostal space $=0$ points; more than 3 distinctive $B$ lines or 1 coalescent B line $=1$ point, quantifying interstitial inflammation or small bronchiectasis (Figure 1) confirmed by CT (Figure 2); more than 2 coalescent B lines = 2 points, suggestive of alveolointerstitial inflammation or mucus plugging with loss of aeration; either bronchial wall thickening or subpleural consolidation $<1 \mathrm{~cm}=3$ points, associated with the absence of A lines quantified either as small atelectasis or cystic bronchiectasis with mucus plugging; subpleural lung consolidation $>1 \mathrm{~cm}$, without bronchogram $=4$ points; quantified atelectasis (Figure 3)/consolidation with bronchogram $=5$ points. 
Table 1. LUS-CF artefacts score.

\begin{tabular}{cl}
\hline LUS Artefact & Lung CF Score \\
\hline $\begin{array}{c}\text { Presence of A lines-normal aspect } \\
\text { Distinctive B lines }<3 / \text { ic space }\end{array}$ & 0 \\
\hline Distinctive B lines $>3 /$ space or 1 coalescent B line & 1 \\
\hline Coalescent B lines $>2 /$ ic space & 2 \\
\hline Consolidation $<1 \mathrm{~cm}$ & 3 \\
\hline Consolidation $>1$ cm, with bronchogram & 4 \\
\hline Atelectasis/consolidation without bronchogram, $>1 \mathrm{~cm}$ & 5 \\
\hline
\end{tabular}

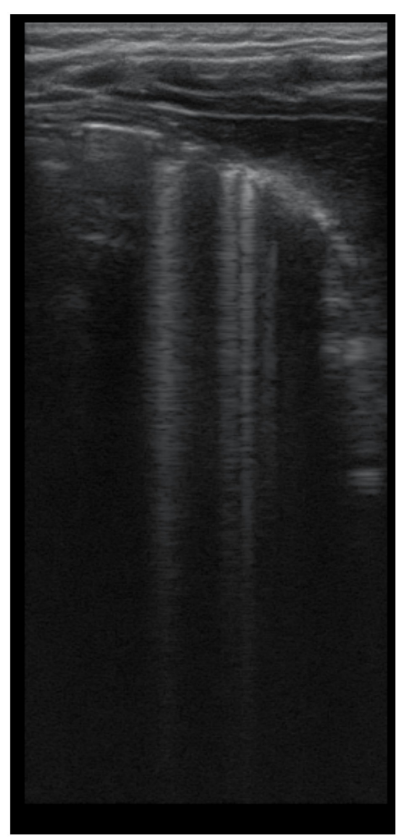

Figure 1. LUS image shows B lines $>3$, LUS score $=2$. The corresponding CT image (Figure 2) shows bronchiectasis.

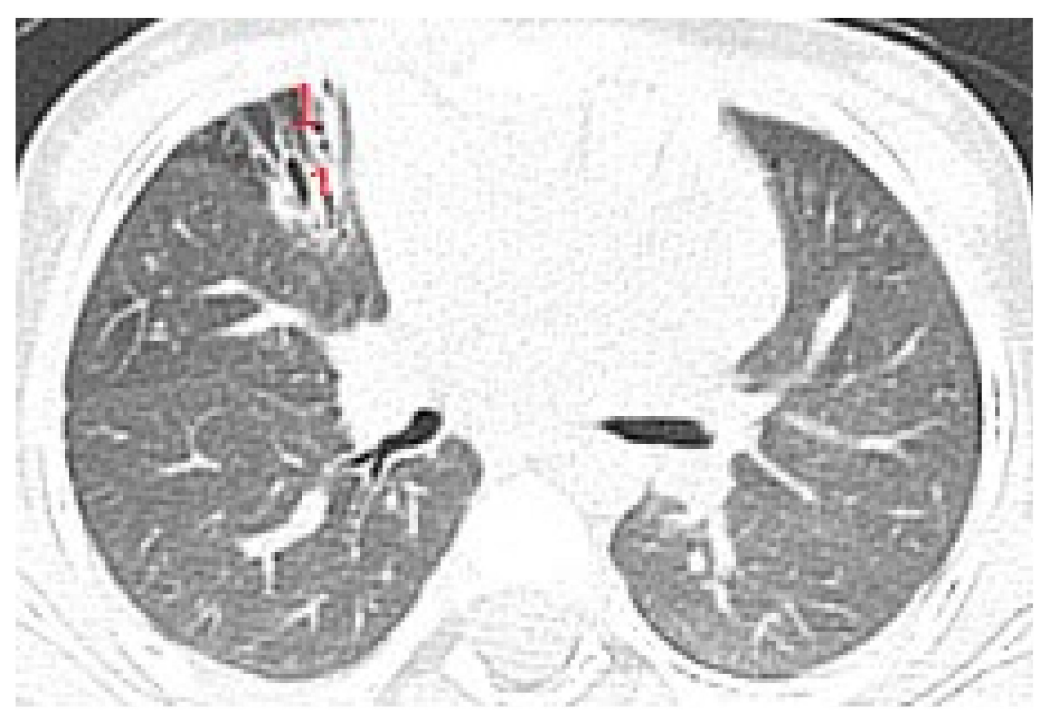

Figure 2. CT reveals (1) peripheral cylindrical bronchiectasis with mucus plugging. 


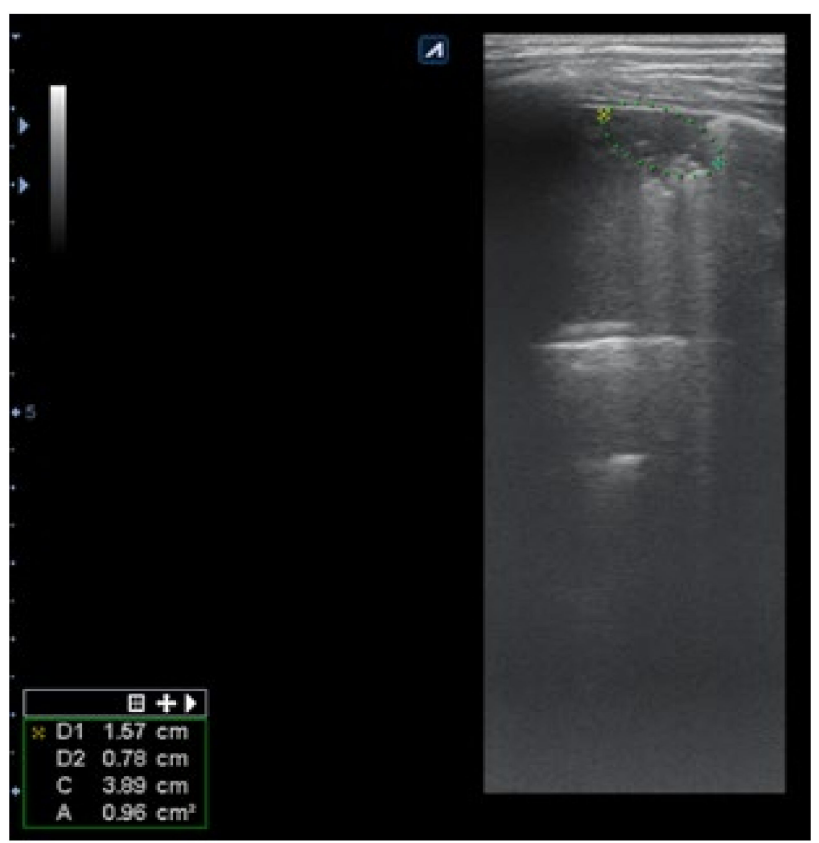

Figure 3. LUS: subpleural consolidation of $1.75 \mathrm{~cm} / 0.78 \mathrm{~cm}$, surface of $0.96 \mathrm{~cm}^{2}$ (score 4), without bronchogram, with adjacent B lines (2 points); A lines also present, examination of left posterior hemithorax.

Subpleural consolidation were detected by CT scan (Figure 4) in 33.3\% of patients, and confirmed by LUS in $31.5 \%$ (Figure 3 ).

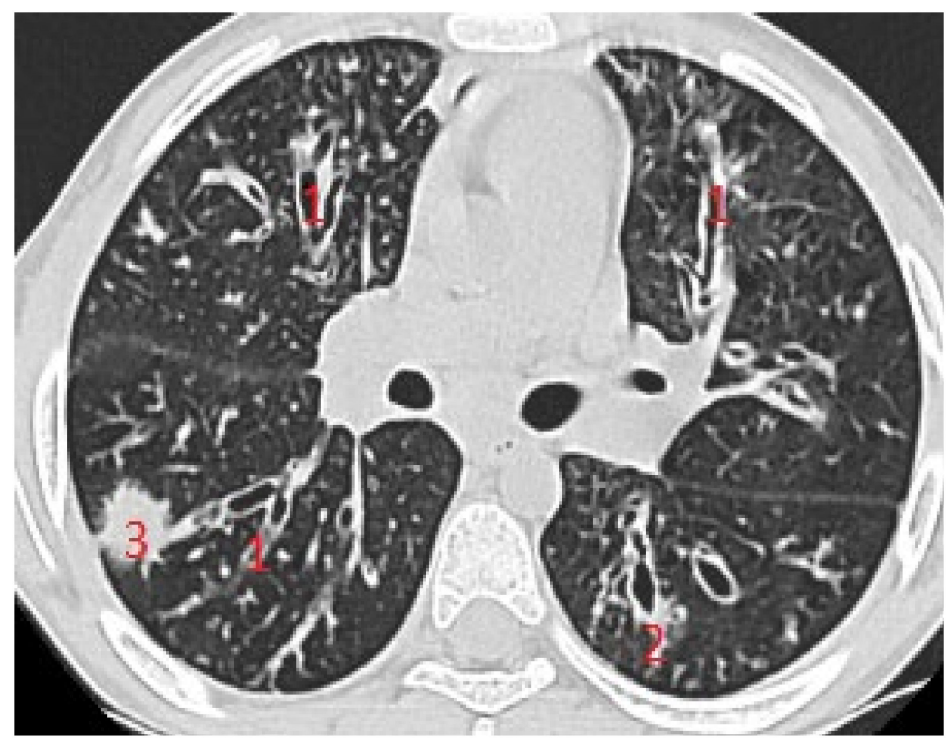

Figure 4. CT scan of the same patient, with various types of bronchiectasis: (1) cylindrical bronchiectasis with moderate bronchial wall thickening; (2) varicose bronchiectasis; and (3) a round/spiculated consolidation, corresponding to previous LUS consolidation. CT score $=62$.

The right hemithorax of the same patients revealed the presence of $B$ lines for cylindrical bronchiectasis via LUS (Figure 5). 


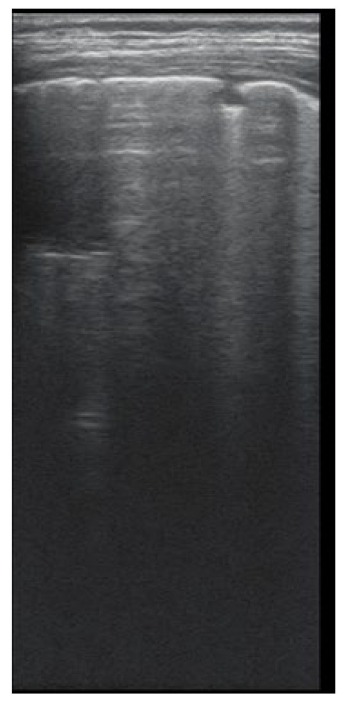

Figure 5. LUS: coalescent B lines, with a very small subpleural consolidation (2 points) and 2 coalescent B lines (2 points), corresponding to mucus-filled varicose bronchiectasis; examination of the same patient's right posterior hemithorax.

The calculation of the score was done by summing up the lesions detected in the six zones of every corresponding hemithorax. The mean LUS score was $5.88 \pm 5.4 \mathrm{SD}$, ranging from 0-21. The mean CT score was $38.14 \pm 11.1$, consistent with the moderate structural lung damage, ranging from 4 points to a maximum of 82 points.

\subsection{Spearman's Correlation Test}

Taking into consideration the fact that our data have a nonparametric distribution, we used Spearman's rho coefficient in order to evaluate the correlation between LUS score and CT score, FEV1, FEF 25-75, and LCI.

\subsubsection{LUS-CF Score and CT Score}

The LUS-CF score had a very strong correlation with the CT score of $r s=0.87$, showing important statistical significance $(p=0.000)$, suggesting a good reliability of the LUS-CF score in the evaluation of $\mathrm{CF}$ lung parenchymal deterioration.

We divided the patients according to their CT score: mild disease (0-33), moderate disease (34-66), and severe disease (> 67) (Figure 6). The correlation in patients with mild disease, expressed by an LUS-CF score from 0 to 7 , was weak $(\mathrm{rs}=0.439)(p=0.014)$, while in patients with moderate disease, the correlation coefficient strongly increased to $\mathrm{rs}=0.57$, with good statistical significance $(p=0.01)$. By applying the Kruskal-Wallis test, we found a statistical difference between the median LUS values in the categories of the CT score $(\mathrm{H}=39.845, p=0.000$; Table 2).

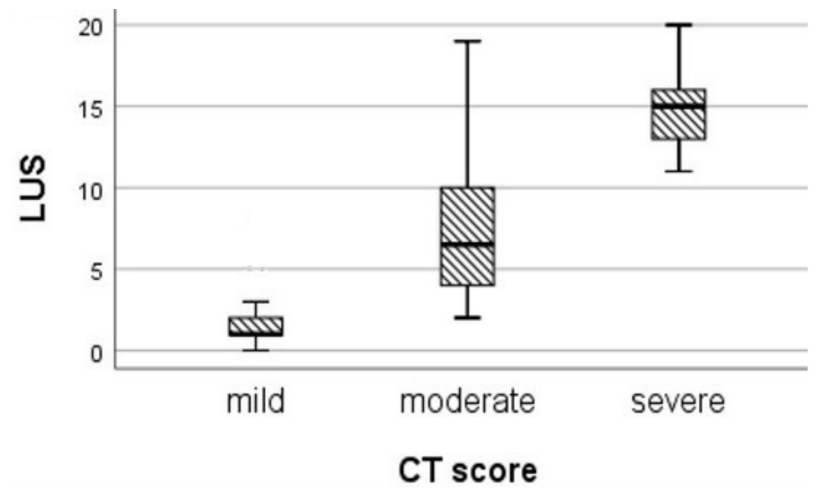

Figure 6. Median LUS scores in patients classified by CT score. 
Table 2. Median LUS scores in CT score categories.

\begin{tabular}{cccccc}
\hline & $\begin{array}{c}\text { Mild CT } \\
\text { Score }\end{array}$ & $\begin{array}{c}\text { Moderate } \\
\text { CT Score }\end{array}$ & Severe CT Score & H & $p$ \\
\hline LUS & & & & & \\
Median (IQR) & $1(1 ; 2)$ & $6.5(4 ; 11)$ & $15(12.75 ; 16.5)$ & 39.845 & 0.000 \\
Mean of rank & 16.07 & 38.14 & 50.05 & & \\
\hline
\end{tabular}

In patients with important structural lung damage (Figure 7), quantified as severe disease, expressed by a CT score $>66$, the correlation was strong $(\mathrm{rs}=0.83)$, with statistical significance $(p=0.002)$.

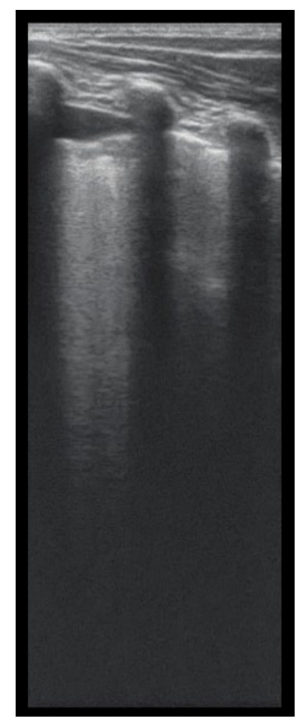

(A)

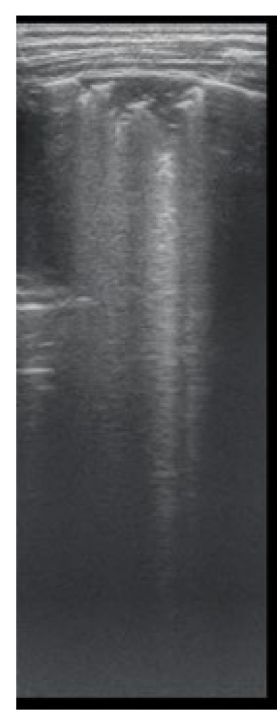

(B)

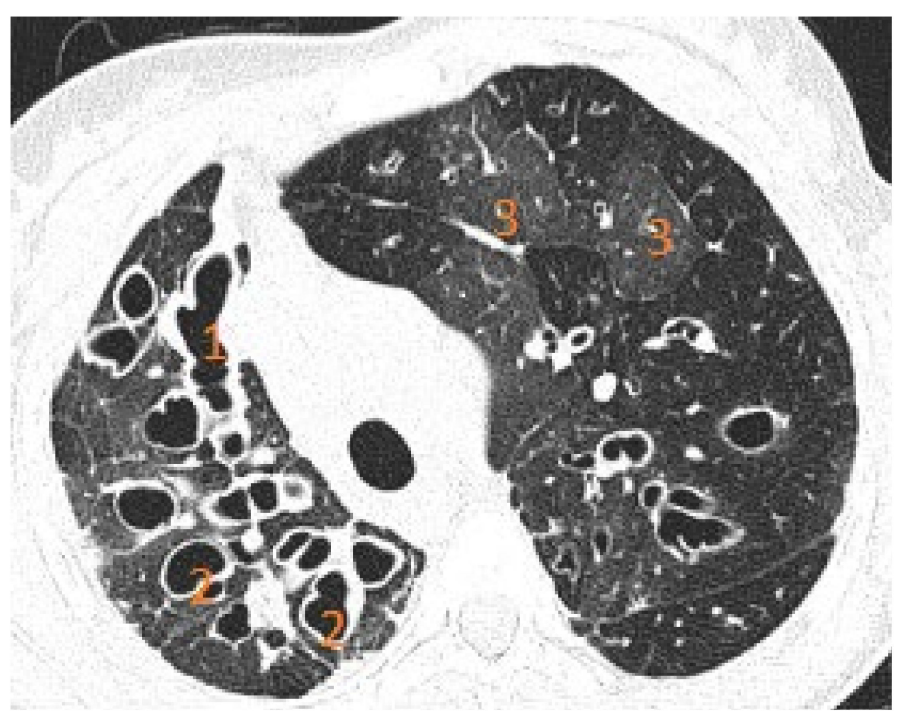

(C)

Figure 7. (A) LUS: coalescent B lines, erased A profile, loss of aeration, left hemithorax. (B) LUS image with subpleural consolidations, coalescent B lines, and left hemithorax. (C) CT scan of the same patients: (1) varicose bronchiectasis with middle 1/3 of lung extended and moderate bronchial wall thickening; (2) saccular bronchiectasis with mild and moderate wall thickening; and (3) zones with increased attenuation of pulmonary parenchyma (alveolar infiltrates).

\subsubsection{LUS-CF Score with Lung Function Parameters}

Evaluating the relationship between the LUS score and LCI, the Spearman's correlation coefficient $\mathrm{rs}=0.8$ revealed a strong, statistically significant correlation $(p=0.000)$-an encouraging significant association between the structural lung disease and lung function. Additionally, the relationship between the LUS-CF score and the spirometry parameters was evaluated, and a strong negative correlation was found with FEV1 rs $=-0.65(p=0.000)$ and FEF $25-75$ rs $=-0.542(p=0.000)$.

\subsection{LUS Sensitivity and Specificity}

The assessment of LUS sensitivity and specificity in bronchiectasis detection varied with the form of bronchiectasis: for cylindrical bronchiectasis(Figure 8A,B), LUS Se $=77.7 \%$, $\mathrm{Sp}=9 \%, \mathrm{PPV}=80.7 \%$, and NPV $=76.9 \%$, while for saccular bronchiectasis (Figure 7), a moderate $\mathrm{Se}=68.4 \%$, with good $\mathrm{Sp}=94.9 \%$, PPV $=88.8 \%$, and NPV $=94.7 \%$ were found. 


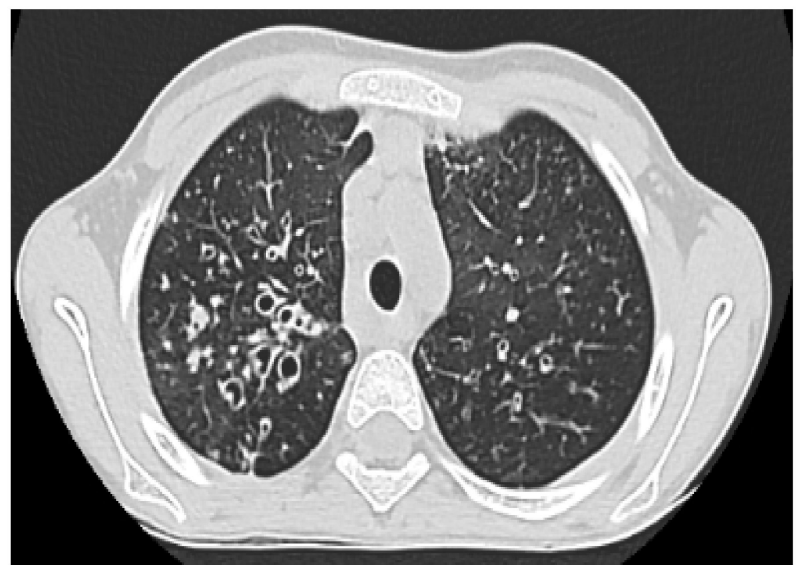

(A)

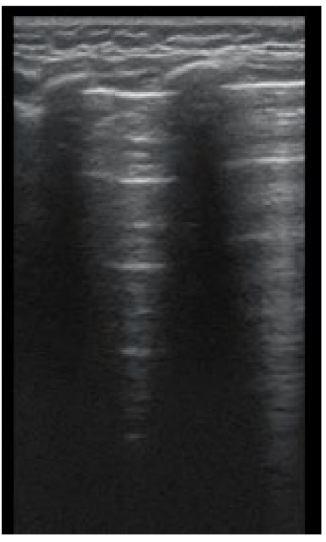

(B)

Figure 8. (A) CT scan: cylindrical bronchiectasis with mucus plugs (B) LUS: A lines, normal LUS aspect, score $=0$.

As for varicose bronchiectasis (Figure $9 \mathrm{~A}, \mathrm{~B}$ ), a very low $\mathrm{Sp}=25 \%$ and NPV $=16.6 \%$ were calculated, with a satisfactory PPV $=88.8 \%$ and $\mathrm{Se}=68.4 \%$.

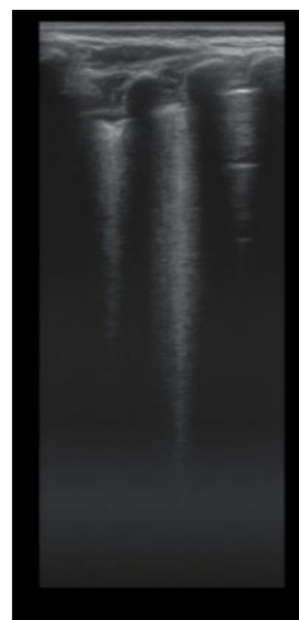

(A)

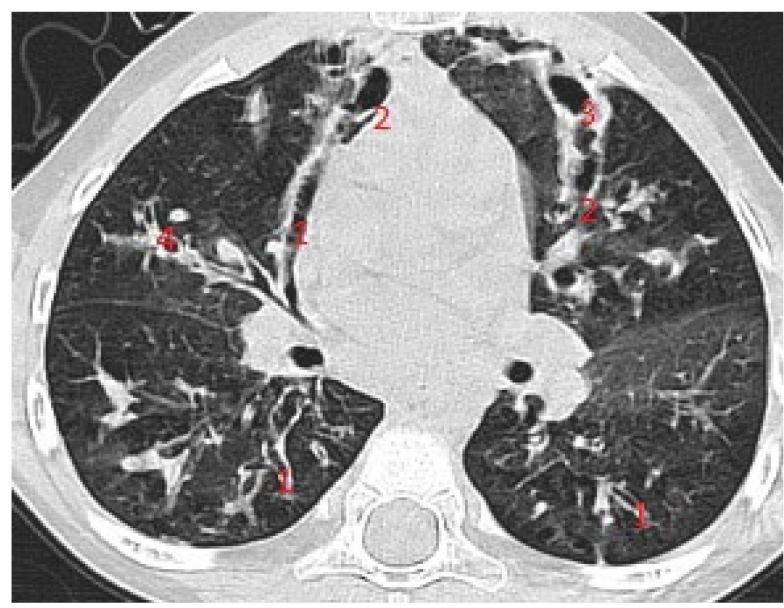

(B)

Figure 9. (A) LUS: coalescent B lines, loss of A lines. (B) CT image: (1) cylindrical bronchiectasis with moderate wall thickening; (2) varicose bronchiectasis; (3) saccular bronchiectasis with moderate wall thickening; and (4) several bronchiectasis with mucus plugging.

The results regarding atelectasis (Figures 10 and 11) and consolidation detection were significant. LUS showed good sensitivity and specificity in detecting atelectasis (Figure 10) $\mathrm{Se}=83.7 \%, \mathrm{Sp}=94.5 \%, \mathrm{PPV}=92.5 \%, \mathrm{NPV}=72.3 \%$-and consolidations-Se $=94.4 \%$, $\mathrm{Sp}=93.02 \%, \mathrm{PPV}=89.4 \%, \mathrm{NPV}=97.3 \%$.

As for bronchial thickening, low sensitivity and specificity were found: $\mathrm{Se}=31.7 \%$, $\mathrm{Sp}=35.2 \%, \mathrm{PPV}=54.1, \mathrm{NPV}=14.2 \%$.

We could not calculate the reliability of LUS for air trapping or for mucus plugging because of a lack of specific artefacts. 


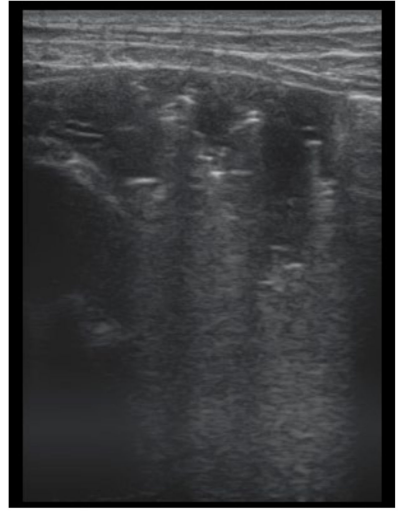

(A)

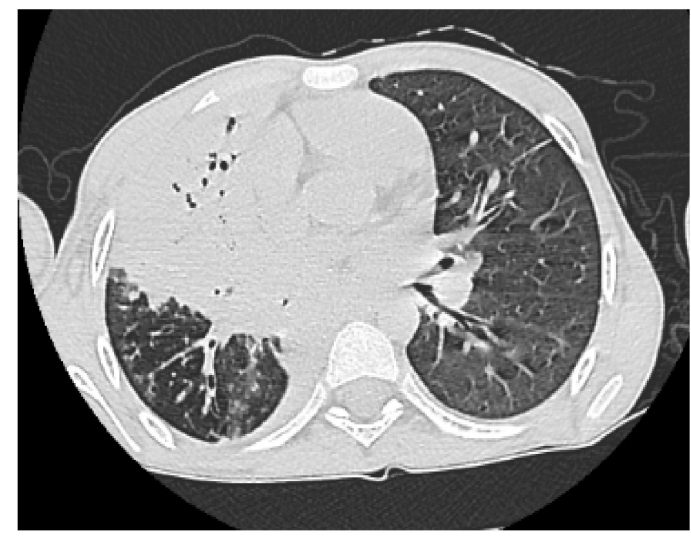

(B)

Figure 10. (A) LUS image of atelectasis, hypoechoic image with air inside. (B) CT scan reveals atelectasis, bronchiectasis, and partial bronchogram.

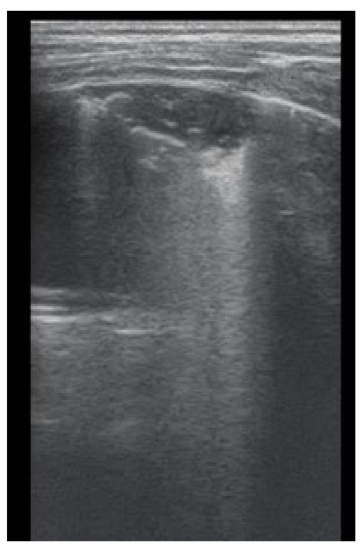

(A)
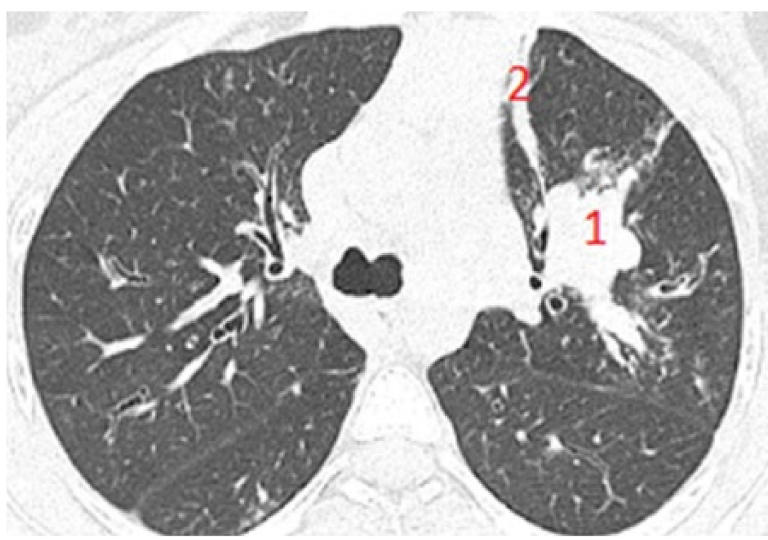

(B)

Figure 11. (A) LUS image of atelectasis, consolidation without bronchogram. (B) CT exam illustrates (1) a peribronchovascular consolidation without air bronchogram, and (2) a lamellar (band) atelectasis.

\section{Discussion}

The literature on LUS-CF is limited, as CF is a rare disease with few patients, and a small number of specialists in LUS.

In this study, we found that the LUS-CF score is a valuable instrument not only to reveal the presence and quantification of parenchymal injury in CF, but also for expressing the relationship with lung clearance index - the most accurate $\mathrm{CF}$ functional parameter.

LUS can show many ultrasound abnormalities, such as B lines, pleural line abnormalities, important consolidations, and atelectasis. In addition to its diagnostic practicality, LUS also seems to be effective for detection of exacerbations [40], showing good correlation with lung function $[17,41]$. Few studies have investigated the significance of LUS in CF $[15-17,40,41]$, but emerging evidence remains to be shown. Our study is the first to describe the LUS artefacts corresponding to CT lung lesions, quantifying all lung injuries potentially detected by LUS. Furthermore, this is the first study to evaluate the relationship between lung structural damage expressed by LUS scoring and functional issues expressed by LCI, as we previously noted this correlation between structure and function [41].

The first study that presented the CF lung ultrasound artefacts in cystic fibrosis described the presence of interstitial syndrome, bronchiectasis, alveolar consolidation, and pleural signs, but was published only in abstract, by our group [41]. Strzelczuk-Judka subsequently reported the CF-USS (cystic fibrosis ultrasound score), which evaluated the presence and extent of pleural irregularities, focal or coalescent "lung rockets" B lines, 
subpleural consolidations, and pleural fluid, showing a positive correlation with ChrispinNorman CXR scoring systems ( $\mathrm{r}=0.52, p=0.0002)$ [16]. As in our study, they acknowledged an important limitation in the inability to visualize the respiratory airway deterioration, e.g., bronchiectasis and mucus plugs.

Peixoto et al. reported a score that included A pattern and B pattern and $\mathrm{C}$ pattern (consolidation), stratifying patients into A profile, B profile, C profile, or mixed profiles compared to CT, and reported a good correlation between LUS and CT [17]. The presence of pleural effusion was not scored (declared as very rare), nor were the pleural irregularities (only describing the finding) [17], similar to our study. The choice of excluding pleural effusion from our LUS-CF score was based on the fact that pleural effusion is not a specific feature of CF.

Hassanzad et al. noted in LUS the presence of pleural thickening, atelectasis, air bronchogram, B lines, and consolidation, compared the findings with corresponding CXR and HRCT, and evaluated the diagnostic performance of LUS and CXR for every artefact, with satisfactory results [40]. Similar to our discoveries, a good diagnostic performance for the detection of consolidation was reported in this paper.

Regarding pleural irregularities, Strzelczuk-Judka noted pleural irregularities similar to bronchiolitis in one patient [16]; Peixoto described this feature, but did not quantify it [17]. We did not take into consideration the presence of the pleural irregularities for this 2016 starting study, because CT did not show a specific corresponding finding; therefore, we considered it normal appearance at the time of our LUS-CF score's development. Neither of the others studies stated that pleural irregularities or thickening would correspond to a specific modification in CT.

With previous experience of our group on LUS in CF [41], we noted that LUS artefacts may quantify different pathological expression. As exemplified in the results of the present paper, B lines can also quantify interstitial inflammation or small bronchiectasis, as confirmed by CT in our study. Similarly, the coalescent B lines can be suggestive of alveolo-interstitial inflammation or mucus plugging with loss of aeration, or bronchial wall thickening. We observed that subpleural consolidation with absence of A lines quantified small atelectasis, but also cystic bronchiectasis filled with mucus. The lack of specificity for mentioned artefacts led us to be cautious in asserting the specificity of LUS for CF lung disease. However, the LUS-CF score showed a very good correlation with CT, as mentioned, and was highly sensitive in detecting parenchymal abnormalities. Other studies suggest the role of LUS in CF exacerbations [42], showing its superiority to CXR in terms of the detection of consolidations, pleural effusion and irregularities compared to CT examination [40] in exacerbations of CF in patients. In previously published papers, a good correlation was found between the LUS and Chrispin-Norman CXR score [16], and also with the modified Bhalla CT score [17], which is also reflected in our own findings.

Our findings reveal a very strong positive linear correlation between the LUS-CF score and LCI ( $\mathrm{rs}=0.87, p=0.000)$, suggesting the reliability of the LUS-CF score in the detection of functional impairment in CF patients. The correlation coefficient between LUS and LCI was superior to the correlation with spirometry parameters, which can be explained by the increased sensitivity of LCI in the detection of lung function impairment and the specificity of the LUS-CF score that included all B line spectra, consolidations with or without bronchiectasis, and loss of aeration.

A strong negative correlation was found with CF-specific parameters for obstructions such as FEV1 ( $\mathrm{rs}=-0.65(p=0.000)$ and FEF 25-75 ( $\mathrm{ss}=-0.542(p=0.000)$. These findings are similar to those of another study that evaluated LUS patterns in CF, and which showed a correlation with lung function expressed by pre-BD FVC $r=0.538$ and pre-BD FEV1 $\mathrm{r}=0.536$ [17].

Descriptive data on LUS artefacts in a number of conditions have been published, but the development of LUS scores has opened the door to objective evaluation. These studies show a correlation between LUS scores and inflammation [29], lung function in CF, and even mortality prognosis [43] in several diseases. 
The reliability of LUS in CF lung disease is sustained by its very good sensitivity and specificity in detecting consolidations ( $\mathrm{Se}=94.4 \%, \mathrm{Sp}=93.02 \%)$, a reliable positive predictive value of $89.4 \%$, and an important NPV $=97.3 \%$. LUS detected atelectasis with a good sensitivity of $83.7 \%$ and a significant specificity of $94.5 \%$. The positive predictive value was important for atelectasis (PPV $=92.5 \%$ ), and NPV $=72.3 \%$. These findings are similar to those of other studies $[16,17,40]$.

As for LUS sensitivity and sensibility in bronchiectasis, detection varied with the type of bronchiectasis, with a decent sensitivity for detecting cylindrical bronchiectasis (77.7\%) and saccular bronchiectasis ( $\mathrm{Se}=68.4 \%, \mathrm{Sp}=94.9 \%$ ), but a lower specificity of $9 \%$ for cylindrical bronchiectasis, explained by the fact that they were quantified by B lines which, as stated, suggest several diseases [44].

The results regarding atelectasis detection were significant; LUS showed good sensibility and specificity in detecting atelectasis: $\mathrm{Se}=83.7 \%, \mathrm{Sp}=94.5 \%, \mathrm{PPV}=92.5 \%$, $\mathrm{NPV}=72.3 \%$. As for bronchial thickening, low sensitivity and specificity were found: $\mathrm{Se}=31.7 \%, \mathrm{Sp}=35.2 \%, \mathrm{PPV}=54.1, \mathrm{NPV}=14.2 \%$.

This study has a few limitations. Lung ultrasound was performed by a single practitioner, and while the number of patients included in the study was satisfactory, it is possible that a larger study would have offered more consistency. Another limitation is that important artefacts such as air trapping/emphysema-one of the premature signs of lung damage in $\mathrm{CF}$-has no US correspondence. No artefact for emphysema quantification was found, nor has any been published previously. Furthermore, small mucus plugging or mild bronchial wall thickening was not detected by LUS, as no LUS artefacts for these changes have been identified to date.

An additional important issue is related to the lack of specificity of B lines, which can quantify a number of diseases [44], ranging from interstitial lung disease or bronchiolitis to acute respiratory distress syndrome or bronchiectasis [45]. We specifically quantified different extensions of bronchiectasis (i.e., cylindrical, varicose, cystic) by the presence of B lines to increase the accuracy of our study, and we eliminated the patients in exacerbations in order to decrease the misinterpretations of interstitial inflammation that can occur in exacerbations with bronchiectasis. As bronchiectasis is the hallmark of CF lung disease, special attention was given to its detection via LUS, and we found LUS to be reliable for the detection of bronchiectasis - a finding that is similar to those of recent studies in adult pathology [46]. The presence of more than three individual B lines per intercostal space, in longitudinal sections, detected before CT examination, suggested certain structural changes. In some of the patients, the above-mentioned studies quantified bronchiectasis to different degrees; in others, bronchial wall thickening or small mucus plugging-specific features identified on CT; therefore, they are not specific to a single lesion, but their presence definitely indicates a lung lesion.

The validation of our score is expressed by the strong correlation found with the modified Bhalla CT score-especially in severely altered lung diseases. However, CT remains the gold standard for CF lung morphological evaluation, as it can accurately detect alterations that are not encryptable via LUS so far, such as air trapping and mucus plugging, considering that bronchiectasis and air trapping are important validated outcome parameters [47].

Even if HRCT is the gold standard for lung disease in CF, it cannot be used as often as necessary - especially in children diagnosed with $C F$, who require sedation when being exposed to repetitive irradiating scans, even if a low dosage is used.

LUS can be a reliable instrument for screening and monitoring children with $\mathrm{CF}-$ especially in the advanced forms of the disease-reducing the levels of exposure to CT radiation.

The strength of this study is the presentation of the LUS-CF score as a non-invasive and reliable tool for the screening of advanced structural damage in $\mathrm{CF}$. 


\section{Conclusions}

LUS in the monitoring of CF patients' lung disease is reliable for advanced lung disease and for lesions of moderate severity, but for the detection of early changes LUS is not a consistent method of lung investigation, with CT remaining the gold standard.

Our study has shown that the LUS-CF score is a parameter that can be used for an associated evaluation, and can play a complementary role in the diagnosis and monitoring of CF lung disease in children.

Author Contributions: Conceptualization, I.M.C.; data curation, I.M.C., E.R.S., A.M.M., and D.L.M.; formal analysis, I.M.C., L.L.P., M.D., E.R.S., M.S.M., A.M.M., and D.L.M.; investigation, M.D., E.R.S., M.S.M., A.M.M., and D.L.M.; methodology, L.L.P., M.D., and E.R.S.; project administration, L.L.P. and M.S.M.; software, A.M.M.; supervision, L.L.P. and D.L.M.; validation, A.M.M. and D.L.M.; visualization, I.M.C., L.L.P., M.D., E.R.S., M.S.M., and A.M.M.; writing—original draft preparation, I.M.C.; writing—review and editing, I.M.C., L.L.P., M.D., M.S.M., and D.L.M. All authors have read and agreed to the published version of the manuscript.

Funding: This research received no external funding.

Institutional Review Board Statement: This study was conducted according to the guidelines of the Declaration of Helsinki, and approved by the Ethics Committee of Clinical County Hospital Timisoara, Romania (No.8/11.01.2016).

Informed Consent Statement: Informed consent was obtained from all subjects involved in the study; patients and their parents signed the informed consent and agreed to the study.

Data Availability Statement: Data available on request from the corresponding author.

Acknowledgments: The authors acknowledge the colleagues from the Pediatric Clinic Pulmonology Unit and National Cystic Fibrosis Center of Clinical County Hospital who participated in the care of the patients.

Conflicts of Interest: The authors declare no conflict of interest.

\section{References}

1. Bell, S.C.; Mall, M.A.; Gutierrez, H.; Macek, M.; Madge, S.; Davies, J.C.; Burgel, P.-R.; Tullis, E.; Castaños, C.; Castellani, C.; et al. The future of cystic fibrosis care: A global perspective. Lancet Respir. Med. 2020, 8, 65-124. [CrossRef]

2. Goss, C.H. Acute Pulmonary Exacerbations in Cystic Fibrosis. Semin. Respir. Crit. Care Med. 2019, 40, 792-803. [CrossRef] [PubMed]

3. Cleveland, R.H.; Neish, A.S.; Zurakowski, D.; Nichols, D.P.; Wohl, M.E.B.; Colin, A.A. Cystic fibrosis: A system for assessing and predicting progression. Am. J. Roentgenol. 1998, 170, 1067-1072. [CrossRef]

4. Tiddens, H.A.W.M. Chest computed tomography scans should be considered as a routine investigation in cystic fibrosis. Paediatr. Respir. Rev. 2006, 7, 202-208. [CrossRef] [PubMed]

5. Pereda, M.A.; Chavez, M.A.; Hooper-Miele, C.C.; Gilman, R.H.; Steinhoff, M.C.; Ellington, L.E.; Gross, M.; Price, C.; Tielsch, J.M.; Checkley, W. Lung ultrasound for the diagnosis of pneumonia in children: A meta-analysis. Pediatrics 2015, 135, 714-722. [CrossRef] [PubMed]

6. Volpicelli, G.; Elbarbary, M.; Blaivas, M.; Lichtenstein, D.A.; Mathis, G.; Kirkpatrick, A.W.; Melniker, L.; Gargani, L.; Noble, V.E.; Via, G.; et al. International evidence-based recommendations for point-of-care lung ultrasound. Intensive Care Med. 2012, 38, 577-591. [CrossRef]

7. Esposito, S.; Papa, S.S.; Borzani, I.; Pinzani, R.; Giannitto, C.; Consonni, D.; Principi, N. Performance of lung ultrasonography in children with community-acquired pneumonia. Ital. J. Pediatrics 2014, 40, 37. [CrossRef]

8. Heuvelings, C.C.; Bélard, S.; Familusi, M.A.; Spijker, R.; Grobusch, M.P.; Zar, H.J. Chest ultrasound for the diagnosis of paediatric pulmonary diseases: A systematic review and meta-analysis of diagnostic test accuracy. Br. Med. Bull. 2019, 129, 35-51. [CrossRef]

9. Kharasch, S.; Duggan, N.M.; Cohen, A.R.; Shokoohi, H. Lung ultrasound in children with respiratory tract infections: Viral, bacterial or covid-19? A narrative review. Open Access Emerg. Med. 2020, 12, 275-285. [CrossRef]

10. Urbankowska, E.; Krenke, K.; Drobczyński, Ł.; Korczyński, P.; Urbankowski, T.; Krawiec, M.; Kraj, G.; Brzewski, M.; Kulus, M. Lung ultrasound in the diagnosis and monitoring of community acquired pneumonia in children. Respir. Med. 2015, 109, 1207-1212. [CrossRef]

11. Heuvelings, C.C.; Bélard, S.; Andronikou, S.; Lederman, H.; Moodley, H.; Grobusch, M.P.; Zar, H.J. Chest ultrasound compared to chest X-ray for pediatric pulmonary tuberculosis. Pediatric Pulmonol. 2019, 54, 1914-1920. [CrossRef] [PubMed] 
12. Daswani, D.D.; Shah, V.P.; Avner, J.R.; Manwani, D.G.; Kurian, J.; Rabiner, J.E. Accuracy of Point-of-care Lung Ultrasonography for Diagnosis of Acute Chest Syndrome in Pediatric Patients with Sickle Cell Disease and Fever. Acad. Emerg. Med. 2016, 23, 932-940. [CrossRef] [PubMed]

13. Sansone, F.; Attanasi, M.; di Filippo, P.; Sferrazza Papa, G.F.; di Pillo, S.; Chiarelli, F. Usefulness of lung ultrasound in paediatric respiratory diseases. Diagnostics 2021, 11, 1783. [CrossRef] [PubMed]

14. Ciuca, I.M.; Pop, L.L. 145 Lung ultrasound in CF children's exacerbation-one center experience. J. Cyst. Fibros. 2015, 14 (Suppl. 1), S95. [CrossRef]

15. Ciuca, I.M.; Dediu, M.; Pop, L.L. Lung clearance index and lung ultrasound in cystic fibrosis children. Eur. Respir. J. 2018, 52, OA4988.

16. Strzelczuk-Judka, L.; Wojsyk-Banaszak, I.; Zakrzewska, A.; Jończyk-Potoczna, K. Diagnostic value of chest ultrasound in children with cystic fibrosis-Pilot study. PLoS ONE 2019, 14, e0215786. [CrossRef]

17. Peixoto, A.O.; Al Marson, F.; Dertkigil, S.S.J.; Dertkigil, R.P.; Souza, T.H.; Fraga, A.M.A.; Ribeiro, A.F.; Toro, A.A.; Ribeiro, J.D. The use of ultrasound as a tool to evaluate pulmonary disease in cystic fibrosis. Respir. Care 2020, 65, 293-303. [CrossRef]

18. Folescu, T.W.; de Andrade Marques, E.; Boechat, M.C.B.; Daltro, P.; Higa, L.Y.S.; Cohen, R.W.F. High-resolution computed tomography scores in cystic fibrosis patients colonized with Pseudomonas aeruginosa or Staphylococcus aureus. J. Bras. Pneumol. 2012, 38, 41-49. [CrossRef]

19. Brody, A.S.; Sucharew, H.; Campbell, J.D.; Millard, S.P.; Molina, P.L.; Klein, J.S.; Quan, J. Computed tomography correlates with pulmonary exacerbations in children with cystic fibrosis. Am. J. Respir. Crit. Care Med. 2005, 172, 1128-1132. [CrossRef]

20. Bhalla, M.; Turcios, N.; Aponte, V.; Jenkins, M.; Leitman, B.; McCauley, D.I.; Naidich, D. Cystic fibrosis: Scoring system with thin-section CT. Radiology 1991, 179, 783-788. [CrossRef]

21. Rosenow, T.; Oudraad, M.C.J.; Murray, C.P.; Turkovic, L.; Kuo, W.; de Bruijne, M.; Ranganathan, S.C.; Tiddens, H.A.W.M.; Stick, S. PRAGMA-CF. A Quantitative Structural Lung Disease Computed Tomography Outcome in Young Children with Cystic Fibrosis. Am. J. Respir. Crit. Care Med. 2015, 191, 1158-1165. [CrossRef] [PubMed]

22. Soldati, G.; Smargiassi, A.; Inchingolo, R.; Buonsenso, D.; Perrone, T.; Briganti, D.F.; Perlini, S.; Torri, E.; Mariani, A.; Mossolani, E.E.; et al. Proposal for international standardization of the use of lung ultrasound for COVID-19 patients; a simple, quantitative, reproducible method. J. Ultrasound Med. Off. J. Am. Inst. Ultrasound Med. 2020, 39, 1413-1419.

23. Dargent, A.; Chatelain, E.; Kreitmann, L.; Quenot, J.-P.; Cour, M.; Argaud, L.; The COVID-LUS Study Group. Lung ultrasound score to monitor COVID-19 pneumonia progression in patients with ARDS. PLoS ONE 2020, 15, e0236312. [CrossRef]

24. Volpicelli, G.; Lamorte, A.; Villén, T. What's new in lung ultrasound during the COVID-19 pandemic. Intensive Care Med. 2020, 46, 1445-1448. [CrossRef] [PubMed]

25. Linsalata, G.; Okoye, C.; Antognoli, R.; Guarino, D.; Ravenna, V.; Orsitto, E.; Calsolaro, V.; Monzani, F. Pneumonia Lung Ultrasound Score (PLUS): A New Tool for Detecting Pneumonia in the Oldest Patients. J. Am. Geriatr. Soc. 2020, 68, 2855-2862. [CrossRef]

26. Costamagna, A.; Pivetta, E.; Goffi, A.; Steinberg, I.; Arina, P.; Mazzeo, A.T.; Del Sorbo, L.; Veglia, S.; Davini, O.; Brazzi, L.; et al. Clinical performance of lung ultrasound in predicting ARDS morphology. Ann. Intensive Care 2021, 11, 51. [CrossRef]

27. Bouhemad, B.; Brisson, H.; Le-Guen, M.; Arbelot, C.; Lu, Q.; Rouby, J.-J. Bedside ultrasound assessment of positive end-expiratory pressure-induced lung recruitment. Am. J. Respir. Crit. Care Med. 2011, 183, 341-347. [CrossRef]

28. Enghard, P.; Rademacher, S.; Nee, J.; Hasper, D.; Engert, U.; Jörres, A.; Kruse, J.M. Simplified lung ultrasound protocol shows excellent prediction of extravascular lung water in ventilated intensive care patients. Crit. Care 2015, 19, 36. [CrossRef]

29. Ciuca, I.M.; Dediu, M.; Pop, L.L. Pediatric pneumonia (PedPne) lung ultrasound score and inflammatory markers: A pilot study. Pediatric Pulmonol. 2022, 57, 576-582. [CrossRef]

30. De Martino, L.; Yousef, N.; Ben-Ammar, R.; Raimondi, F.; Shankar-Aguilera, S.; de Luca, D. Lung ultrasound score predicts surfactant need in extremely preterm neonates. Pediatrics 2018, 142, e20180463. [CrossRef]

31. Szymański, P.; Kruczek, P.; Hożejowski, R.; Wais, P. Modified lung ultrasound score predicts ventilation requirements in neonatal respiratory distress syndrome. BMC Pediatrics 2021, 21, 17. [CrossRef] [PubMed]

32. Gustafsson, P.M.; de Jong, P.A.; Tiddens, H.A.W.M.; Lindblad, A. Multiple-breath inert gas washout and spirometry versus structural lung disease in cystic fibrosis. Thorax 2008, 63, 129-134. [CrossRef] [PubMed]

33. Fretzayas, A.; Loukou, I.; Moustaki, M.; Douros, K. Correlation of computed tomography findings and lung function in children and adolescents with cystic fibrosis. World J. Pediatrics 2021, 17, 221-226. [CrossRef] [PubMed]

34. Copetti, R.; Cattarossi, L. Ultrasound diagnosis of pneumonia in children. La Radiol. Med. 2008, 113, 190-198. [CrossRef]

35. Robinson, T.E.; Leung, A.N.; Chen, X.; Moss, R.B.; Emond, M.J. Cystic fibrosis HRCT scores correlate strongly with pseudomonas infection. Pediatric Pulmonol. 2009, 44, 1107-1117. [CrossRef]

36. Miller, M.R.; Hankinson, J.; Brusasco, V.; Burgos, F.; Casaburi, R.; Coates, A.; Crapo, R.; Enright, P.; Van Der Grinten, C.P.M.; Gustafsson, P.; et al. Standardisation of spirometry. Eur. Respir. J. 2005, 26, 319-338. [CrossRef]

37. Quanjer, P.H.; Stanojevic, S.; Cole, T.J.; Baur, X.; Hall, G.L.; Culver, B.H.; Enright, P.L.; Hankinson, J.L.; Ip, M.S.M.; Zheng, J.; et al Multi-ethnic reference values for spirometry for the 3-95-yr age range: The global lung function 2012 equations. Eur. Respir. J. 2012, 40, 1324-1343. [CrossRef]

38. Fuchs, S.I.; Eder, J.; Ellemunter, H.; Gappa, M. Lung clearance index: Normal values, repeatability, and reproducibility in healthy children and adolescents. Pediatr. Pulmonol. 2009, 44, 1180-1185. [CrossRef] 
39. Dancey, C.P.; Reidy, J. Statistics without Maths for Psychology: Using Spss for Windows; Prentice-Hall, Inc.: Edinburgh, UK, 2004.

40. Hassanzad, M.; Kiani, A.; Abedini, A.; Ghaffaripour, H.; Emami, H.; Alizadeh, N.; Zoghi, G.; Hashemi, S.; Velayati, A.A. Lung ultrasound for the diagnosis of cystic fibrosis pulmonary exacerbation. BMC Pulm. Med. 2021, 21, 353. [CrossRef]

41. Ciuca, I.; Dediu, M.; Margau, E.; Pop, L. P114 Lung ultrasound score correlation with lung clearance index. Thorax 2018, 73, A163.

42. Ciuca, I.; Pop, L.; Marc, M.; Oancea, C. How useful is the lung ultrasound in cystic fibrosis? Eur. Respir. Soc. 2016, 48 (Suppl. 60), PA1261.

43. Tana, C.; Ricci, F.; Coppola, M.G.; Mantini, C.; Lauretani, F.; Campanozzi, D.; Renda, G.; Gallina, S.; Lugará, M.; Cipollone, F.; et al Prognostic Significance of Chest Imaging by LUS and CT in COVID-19 Inpatients: The ECOVID Multicenter Study. Respiration 2021, 101, 122-131. [CrossRef] [PubMed]

44. Dietrich, C.F.; Mathis, G.; Blaivas, M.; Volpicelli, G.; Seibel, A.; Wastl, D.; Atkinson, N.S.; Cui, X.-W.; Fan, M.; Yi, D. Lung B-line artefacts and their use. J. Thorac. Dis. 2016, 8, 1356-1365. [CrossRef] [PubMed]

45. Barakat, M. B lines: Role of chest sonography in assessment of bronchiectasis. In: 13 Imaging. Eur. Respir. Soc. 2016, 48, PA3797.

46. Ghany, M.F.A. Transthoracic ultrasound in the diagnosis of bronchiectasis: Is it valuable? Egypt. J. Bronchol. 2019, 13, 303-308. [CrossRef]

47. Loeve, M.; Krestin, G.P.; Rosenfeld, M.; de Bruijne, M.; Stick, S.M.; Tiddens, H.A. Chest computed tomography: A validated surrogate endpoint of cystic fibrosis lung disease? Eur. Respir. J. 2013, 42, 844-857. [CrossRef] 